\title{
A Systematic Review of Economic Analyses Studying Rituximab in R-CHOP Therapy in Patients with Non-Hodgkin Lymphoma
}

\author{
Jenna L. Yoder ${ }^{1}$ and Khalid M. Kamal*,2 \\ ${ }^{1}$ Mylan School of Pharmacy, Duquesne University, Pittsburgh, Pennsylvania, USA \\ ${ }^{2}$ Department of Clinical, Social and Administrative Sciences, Mylan School of Pharmacy, Duquesne University, Pitts- \\ burgh, Pennsylvania, USA
}

\begin{abstract}
:
Objectives: To explore the use of pharmacoeconomic principles through examination of economic evaluations pertaining to the combination of the monoclonal antibody rituximab with conventional CHOP (cyclophosphamide/doxorubicin/ vincristine/prednisone) or CHOP-like chemotherapy regimens in patients with non-Hodgkin lymphoma.

Methods: A literature search was conducted using Evidence-Based Medical Reviews (EBMR), International Pharmaceutical Abstracts (IPA), and Medline databases to identify all economic studies relating to rituximab in combination with CHOP or CHOP-like regimens. The systematic evaluation also utilized the Quality of Health Economic Studies instrument to assess the quality of each study that was included in the final review.

Results: Initially, eight studies were retrieved which included the use of rituximab in non-Hodgkin lymphoma treatment. Of these, four studies were excluded as rituximab was used as a stand-alone treatment option. The remaining four studies involved conventional CHOP therapy versus the combination with rituximab (R-CHOP) in patients with non-Hodgkin lymphoma. One study employed a cost-effectiveness analysis while the remaining three studies used a cost-utility analysis and reported the outcomes in terms of quality-adjusted life years (QALYs).

Conclusions: The cost-effectiveness evaluation illustrated the dominance of R-CHOP over CHOP-alone in terms of both lower costs and increased life years gained. The cost-utility of R-CHOP in terms of costs/QALYs were below the accepted threshold of 50,000 in international monetary units. Through examination of evaluation principles employed, it is found that valid results are highly dependent on the input data, assumptions, and sensitivity analyses. Clinical decisionmakers must take into account specific inclusions of costs relevant to their own practice setting.
\end{abstract}

Keywords: Pharmacoeconomic analysis, CHOP, R-CHOP, rituximab, B-cell non-Hodgkin lymphoma.

\section{INTRODUCTION}

Immunomodulation has emerged as a coveted treatment option in malignancies, a technique that offers meaningful clinical outcomes with fewer side effects than traditional chemotherapeutic regimens [1]. Monoclonal antibodies offer targeted immunomodulation, and these compounds were first produced through the study of cancers related to B-cells. As a result of biologic compound research, rituximab (Rituxan ${ }^{\circledR}$, MabThera $\left.{ }^{\circledR}\right)$ is a chimeric monoclonal antibody raised against $\mathrm{CD} 20$ receptor protein, and is the first to be approved for the treatment of B-cell non-Hodgkin lymphoma [2].

Lymphoma, the most common blood cancer, is a collective term for malignancies related to a dysfunction of the Bcells or T-cells [3]. These cells, or lymphocytes, are responsible for proper immune function. The numerous types of lymphoma can be divided into Hodgkin lymphoma or non-

*Address Correspondence to this author at the Mailing: 314 Bayer Learning Center, Duquesne University, 600 Forbes Ave, Pittsburgh, PA 15282, Tel: 412-396-1926; E-mail: kamalk@duq.edu
Hodgkin lymphoma. Hodgkin lymphoma, or HL, is the rarer of the two types. HL usually afflicts young adults and is characterized by the presence of Reed-Sternberg cells which are not a normal blood component. Non-Hodgkin lymphoma, or NHL, comprises the most common group of the lymphoma malignancies. In 2000-2004, the incidence rate of NHL was 19.3 per 100,000 people in the United States (US) according to the Surveillance, Epidemiology, and End Results (SEER) Program of the National Cancer Institute [4]. Further, it is estimated that NHL will contribute to over 66,000 new cases and more than 19,000 deaths in the US in 2008 [5]. NHL is categorized as either aggressive or indolent, depending on the growth rate of the malignancy. Of the B-cell affected lymphomas, diffuse large-B-cell lymphoma (DLBCL) is the most common and accounts for approximately one-third of all NHL cases [6]. Research related to Bcell lymphomas, particularly DLBCL, has showcased the emerging benefits of novel immunomodulators like rituximab.

Rituximab is a chimeric mouse/human monoclonal antibody which specifically targets the cell-surface protein antigen $\mathrm{CD} 20$ receptor. The $\mathrm{CD} 20$ antigen is found on more than 
95\% of NHL B-cells [7]. In effect, rituximab serves to mark B-cells expressing the CD20 antigen and programs the targeted cells for death, allowing non-malignant B-cells to prosper. Prior to the use of monoclonal antibodies like rituximab, the "gold standard" treatment regimen for DLBCL has been a chemotherapeutic combination of cyclophosphamide, doxorubicin, vincristine, and prednisone - otherwise known as CHOP. With the development of rituximab, investigations of its efficacy in DLBCL ensued, with particular interest in adding rituximab to the standard CHOP therapy (R-CHOP). A randomized trial by Coiffier et al. [8] demonstrated the benefit of R-CHOP over CHOP in terms of disease-free and overall survival rates in patients over age 60 with DLBCL. Another study, the MabThera International Trial (MInT), showed that R-CHOP achieved better complete response rates (20\% difference) and overall survival rates (9\% difference) in young patients with DLBCL versus chemotherapy regimens alone [9]. Furthermore, a comprehensive review of seven randomized controlled trials which looked at the combination of rituximab with chemotherapy in various NHL patient settings revealed better overall survival, greater overall response, and improved disease control when compared to use of chemotherapy alone [10]. Thus, growing acceptance of adding rituximab to standard chemotherapy regimens in NHL patients has arisen. However, the addition of this monoclonal antibody to treatment protocols is not without additional costs. Investigators subsequently have turned their attention toward the pharmacoeconomic side of rituximab therapy, delving into whether or not the added cost of rituximab is worth the demonstrated clinical benefit.

Pharmacoeconomics is, in effect, "balancing the cost with the consequences (outcomes) of pharmaceutical therapies" [11]. Through the use of pharmacoeconomic studies, investigators may explore whether the addition of rituximab to standard chemotherapy regimens, such as R-CHOP, is economically and clinically beneficial. Further, certain techniques used in pharmacoeconomic research allow investigators to incrementally calculate the degree to which rituximab may be a burdensome or dominating therapy [12]. Keeping in mind the accepted therapeutic benefit of rituximab to CHOP therapy in terms of clinical outcomes, the purpose of this review was to collect and analyze the published pharmacoeconomic studies regarding R-CHOP.

The goal of this review was to emphasize the impact of pharmacoeconomic principles through examination of economic studies pertaining to the combination of rituximab with conventional CHOP or CHOP-like therapies in patients with non-Hodgkin's lymphoma. In order to fulfill such a goal, the strengths and weaknesses of the relevant published analyses have been presented with an emphasis on crucial pharmacoeconomic elements, such as proper inclusion of costs, appropriate outcome measures, and the significance of sensitivity analyses.

\section{METHODS}

\section{Literature Search}

Initially, a systematic search of the literature was done in order to identify all economic studies related to rituximab. Using Evidence-Based Medical Reviews (EBMR), Interna- tional Pharmaceutical Abstracts (IPA), and Medline databases, numerous studies were found pertaining to this general topic. Subsequently, the discovered articles were filtered according to the therapeutic indication of rituximab presented in the studies. First, all articles pertaining to the use of rituximab in oncology were identified, yielding approximately one-thousand studies among the three databases. Second, studies were further filtered according to the desired topic of rituximab's combination use with traditional treatment of NHL. Specific search terms included 'rituximab', 'R-CHOP', 'non-Hodgkin lymphoma', 'lymphoma', 'CHOP', 'economic analysis', 'cost', and combinations of these keywords were used for the secondary filtration. Articles were identified from those sorted out pursuant to the inclusion of economic analyses in the study of rituximab with traditional non-Hodgkin's lymphoma treatment. Consequently, eight articles were identified which studied both the economics and clinical use of rituximab in combination with traditional CHOP or CHOP-like therapy in NHL patients.

\section{Inclusion/Exclusion Criteria}

Out of the approximately one-thousand articles initially retrieved under the general topic of the economic evaluation of rituximab, articles were included in the review only if the intention of studying the costs related to rituximab in combination with traditional chemotherapy regimens was outlined transparently in the studies' abstracts. All types of economic analyses were considered, including but not limited to, cost analyses, cost-effectiveness, cost-utility, cost-minimization, and cost-benefit analyses. In order to streamline the review, only articles that studied both costs and the combination use of rituximab with $\mathrm{CHOP}$ or CHOP-like regimens were included. To allow for further comparability, only articles studying NHL patients were included. Due to the specified nature of the review, articles were not further screened according to patient demographics, age, or country of origin. Articles that studied the use of rituximab as a stand-alone treatment were excluded, as the intention of this review was to analyze the pharmacoeconomics of the combination RCHOP and not the monoclonal antibody alone. Articles which included patients other than those with NHL were excluded for purposes of focusing the scope of this analysis. Likewise, studies that did not include economic analyses (those which only looked at the consequences of rituximab in this patient population) were excluded. Economic analyses derived from review articles were also excluded. The preliminary database searches yielded eight studies pertaining to the economic evaluation of R-CHOP in NHL patients. Of the eight studies initially considered, four comprehensively met the inclusion/exclusion criterion and were reviewed (Table 1).

\section{Evaluation of the Quality of Studies Included in the Re- view}

This systematic evaluation utilized the Quality of Health Economic Studies (QHES) instrument to assess the quality of each study that was included in the final review. Each study was rated on 16 criterions that covered the study methods and results. These 16 criterions, chosen by a panel of pharmacoeconomic experts, form the backbone of this comparative tool. The QHES allows users to assign simple point values to reviewed health care economic studies - a 
Table 1. Overview of Included/Excluded Studies

\begin{tabular}{|c|c|c|}
\hline Study Identified & Type of Pharmacoeconomic Evaluation & Inclusion / Exclusion \\
\hline Ferrara et al. 2008 [19] & Cost-effectiveness analysis & $\begin{array}{c}\text { Included: } \\
\text { CHOP versus R-CHOP in non-Hodgkin's } \\
\text { lymphoma patients }\end{array}$ \\
\hline Groot et al. 2005 [22] & Cost-effectiveness analysis & $\begin{array}{c}\text { Included: } \\
\text { CHOP versus R-CHOP in non-Hodgkin's } \\
\text { lymphoma patients }\end{array}$ \\
\hline Hornberger et al. 2005 [26] & Cost-utility analysis & $\begin{array}{c}\text { Included: } \\
\text { CHOP versus R-CHOP in non-Hodgkin's } \\
\text { lymphoma patients }\end{array}$ \\
\hline Best et al. 2005 [25] & Cost-effectiveness analysis & $\begin{array}{c}\text { Included: } \\
\text { CHOP versus R-CHOP in non-Hodgkin's } \\
\text { lymphoma patients }\end{array}$ \\
\hline Knight et al. 2004 & Review article with cost-effectiveness analysis & $\begin{array}{c}\text { Excluded: } \\
\text { Review article }\end{array}$ \\
\hline Agthoven et al. 2005 [23] & Cost analysis & $\begin{array}{l}\text { Excluded: } \\
\text { Rituximab as stand-alone treatment option }\end{array}$ \\
\hline Sweetenham et al. 1999 & Cost-minimization analysis & $\begin{array}{l}\text { Excluded: } \\
\text { Rituximab as stand-alone treatment option }\end{array}$ \\
\hline Malliti et al. 2003 & Cost analysis & $\begin{array}{l}\text { Excluded: } \\
\text { Rituximab as stand-alone treatment option }\end{array}$ \\
\hline
\end{tabular}

weighted valuation based expert analysis and tests accepted by the pharmacoeconomic community [13]. Higher scores on this instrument indicate a better quality. Ofman et al. [14] has published the QHES instrument in a table format, showcasing the validity of the 16-criterion tool along with explanation of the chosen elements and point values based on the available guidelines and expert recommendations.

\section{RESULTS}

The four included studies were similar in that each looked at conventional CHOP therapy versus the combination with rituximab (R-CHOP) in NHL patients. All reviewed studies were assessed with regard to the types of pharmacoeconomic analyses used. One study employed a true cost-effectiveness analysis, evaluating outcomes in terms of life years gained (LYG). Although two of the remaining three studies purported the use of cost-effectiveness analyses, all of the three studies reported outcomes in terms of quality-adjusted life years (QALYs), more specific to cost-utility analyses (Table 2) [15].

\section{Cost-Effectiveness Analysis}

This type of pharmacoeconomic evaluation measures both the costs and effects of two comparators where the effects, or outcomes, are presented in non-monetary or natural units [12]. Cost-effectiveness is used to compare interventions in terms of clinical outcomes, such as life years gained (LYG), with regard to resources used to achieve those outcomes. One of the most useful applications of a costeffectiveness analysis is that it is a technique which allows comparison of competing strategies through an incremental cost-effectiveness ratio. In scenarios where a therapy is more expensive but also more effective than its comparator, the calculation of an incremental ratio allows investigators to determine the additional costs demanded for each additional unit of clinical benefit achieved. A regimen is generally accepted as cost-effective if its incremental ratio is less than 50,000 in international monetary currencies, although this threshold is subject to debate [16]. In essence, costeffectiveness analysis can demonstrate that one strategy is dominant over another if both the costs of that strategy are less and the clinical benefit is greater [17].

Decision analysis is often used in conjunction with a cost-effectiveness evaluation in instances where limited published data is available, uncertainty surrounds the available data, or in cases where it would be unethical to subject actual patients to a trial. Decision analyses also offer the advantage of being able to project short-term published results far into the future - a strategy desirable in scenarios of chronic diseases or otherwise long-term management issues [18]. While cost-effectiveness analyses allow investigators to see the incremental monetary amount required to produce one additional unit of clinical benefit when a therapy is both more expensive and more effective, such an evaluation is complemented by decision analyses which allow investigators to project the course of a disease state over a long time horizon. Combined as one, cost-effectiveness analyses coupled with decision analyses models is a powerful tool in pharmacoeconomic evaluation. 
Table 2. Overview of Studies Using Principles of Economic Analyses in the Evaluation of Combination Rituximab Therapy in NonHodgkin's Lymphoma Patients

\begin{tabular}{|c|c|c|c|c|c|}
\hline Study & $\begin{array}{c}\text { Type of } \\
\text { Evaluation }\end{array}$ & $\begin{array}{l}\text { Alternatives } \\
\text { Compared }\end{array}$ & Outcomes Measured & $\begin{array}{c}\text { Stated* } \\
\text { Perspective }\end{array}$ & Time Horizon \\
\hline Ferrara et al. 2008 & Cost-Effectiveness & \multirow{4}{*}{$\begin{array}{l}\text { Standard CHOP regi- } \\
\text { men versus rituximab- } \\
\text { added R-CHOP regi- } \\
\text { men }\end{array}$} & Life-years gained (LYG) & Payer & 3 years \\
\hline Groot et al. 2005 & $\begin{array}{c}\text { Cost-Effectiveness } \\
(\text { Cost-Utility })^{* *}\end{array}$ & & $\begin{array}{l}\text { Quality-adjusted life years } \\
\text { (QALYs) gained }\end{array}$ & Societal & 15 years \\
\hline Hornberger et al. 2005 & Cost-Utility & & $\begin{array}{l}\text { Quality-adjusted life years } \\
\text { (QALYs) gained }\end{array}$ & Societal & 5 years \\
\hline Best et al. 2005 & $\begin{array}{c}\text { Cost-Effectiveness } \\
\text { (Cost-Utility)* }\end{array}$ & & $\begin{array}{l}\text { Quality-adjusted life years } \\
\text { (QALYs) gained }\end{array}$ & Payer & 15 years \\
\hline
\end{tabular}

*Refers only to the authors' statement of perspective, regardless of actual costs evaluated.

**Studies sometimes refer to cost-utility analyses as a type of cost-effectiveness evaluation; however, QALYs represent a measure of patient utilities, whereas LYG is more specifically a cost-effectiveness measure.

\section{Studies Using Cost-Effectiveness Analysis}

Ferrara and Ravasio [19] employed a decision analysis model to study the cost-effectiveness of R-CHOP with conventional CHOP-only therapy. Only patients younger than age 60 with good-prognosis, as indicated by a low International Prognostic Index, were included in the study. Treatment arms included in the decision tree model were complete response at five months, non-complete response at five months with subsequent rescue therapy, no relapse at three years, or relapse at three years with rescue therapy. Probabilities related to the proposed treatment arms were taken from the MabThera International Trial (MiNT) [9]. The authors looked at clinical outcomes such as percentage of complete response at five months, relapse-free survival at three years, and overall survival at three years for R-CHOP $(n=413)$ and CHOP $(n=410)$ alone.

Stating a payer perspective of the Italian National Health Service, Ferrara and Ravasio intended to include only direct medical costs. Costs incorporated in the study included acquisition costs for the chemotherapy agents in CHOP therapy and for rituximab. Also, rescue therapy costs were incorporated and included chemotherapy drug costs, stem-cell transplantation costs, cost of apheresis, and hospitalization costs. The authors also included other consumed resources' costs such as hematological and biochemical investigations, imaging, and histological analyses. Life years gained (LYG) was calculated at three years and used as the primary efficacy outcome for purposes of the cost-effectiveness evaluation. Mean costs were discounted at a conservative 3\% rate.

Life years gained per patient were found to be 2.697 and 2.517 for R-CHOP and CHOP alone, respectively. Mean total costs per patient were found to be $€ 22,113.44$ for RCHOP and $€ 22,831.17$ for CHOP, thus portraying R-CHOP as the favored treatment in terms of lower cost and greater clinical outcome.

Sensitivity analyses were conducted by the authors on the three event probabilities of complete response at five months, relapse-free survival at three years, and overall three-year survival. These analyses scrutinized the robustness of results by using the least-favorable upper and lower limits of the events' confidence intervals. R-CHOP remained the favored cost-effective therapy when overall three-year survival probabilities were varied. Incremental costeffectiveness ratios were below the acceptable threshold for the varied complete response at five months and relapse-free survival at three years $(€ 16,816$ and $€ 11,967$ in additional costs for R-CHOP per $1 \mathrm{LYG}$, respectively). Further, the authors employed a sensitivity analysis on the assumed 3\% discount rate, confirming the baseline results when the rate was varied from $1 \%$ to $5 \%$. Additional sensitivity analyses performed by Ferrara and Ravasio included a threshold analysis looking at rescue therapy costs, finding that a $6.64 \%$ reduction in the baseline rescue therapy costs would equilibrate the mean total costs per patients among the two treatment groups. Thus, the authors illustrated that the cost of chemotherapeutic rescue therapy has a profound impact on the higher costs in the CHOP-only regimen, showing that an incremental ratio of $€ 12,276$ applies to $\mathrm{R}-\mathrm{CHOP}$ when only stem-cell transplantation is assumed in rescue therapy costs. Ferrara and Ravasio concluded that R-CHOP versus CHOP-only therapy in young, good-prognosis NHL patients was cost-effective in terms of the included direct medical costs and outcomes in LYG from a payer perspective.

\section{Cost-Utility Analysis}

Cost-utility analysis can be thought of as a sub-type of cost-effectiveness evaluation. Where cost-effectiveness considers outcomes in terms of clinical units such as LYG, costutility expresses outcomes in QALYs. A quality-adjusted life year is a single measure that captures both the quality and quantity elements of a health care outcome. The quality element is derived from a subject's value or utility for a given health state [20]. A utility refers to a numerical term in the range of 0.0 to 1.0 , which are associated with the leastpreferred and most-preferred health states, respectively. While the best method for collecting and valuing patient utilities remains debated, it is important to realize that the validity of utility scores is highly dependent on the valuation methods and population settings used. Examples of valuation methods used in deriving utility scores include "the visual analogue scale (VAS), the standard gamble (SG), and the time trade-off (TTO)" [21]. A utility score is linked to the number of years in that state. Accordingly, combining utilities with years, a quality-adjusted life year (QALY) is 
yielded. Like cost-effectiveness analyses, an incremental ratio can be calculated for cost-utility analyses where a monetary cost per one QALY gained is found and compared to an accepted threshold value of 50,000 in international monetary units.

\section{Studies Using Cost-Utility Analysis}

The second study reviewed, a Netherlands investigation by Groot and colleagues [22], illustrates the use of utility analysis as a subset of cost-effectiveness analyses. The study, Cost-Effectiveness of Rituximab (MabThera ${ }^{\circledR}$ ) in DLBCL in the Netherlands, actually is more appropriately termed a cost-utility analysis because results were reported as incremental gains in QALYs. In contrast to the clinical LYG efficacy outcome utilized in the previous study by Ferrara and Ravasio, Groot and colleagues incorporated quality of life into the outcomes comparing R-CHOP versus CHOPonly. While the study similarly investigated these treatment options in NHL patients, the perspective taken in this study was stated to be societal. From a societal perspective, an exhaustive list of all costs associated with treatment (direct, indirect, and intangible) should be considered as society "pays" in all areas of cost categories related to health care. Interestingly, Groot and colleagues purported a societal perspective, but in fact only included direct medical costs such as wholesale drug costs and an assumed average of two specialist visits per year.

A 15 year time horizon was used by Groot and colleagues to follow patients receiving either R-CHOP or CHOP-only regimens. A $4 \%$ discount rate was applied. Markov transition modeling was used where transition between states was derived from hazard rates and event probabilities were based on the Scottish Newcastle Lymphoma Group (SNLG) database for the CHOP-only treatment arm. Event probabilities for the R-CHOP treatment arm were derived from data collected by Coiffer et al. [8]. The authors distinguished young patients from those over age 60, pointing out that response rates are different among these two groups and that older patients usually undergo more intensive rescue therapy, according to a study by Agthoven et al. [23]. Thus, resource use among these two patient populations was differing, based on results of past investigations.

In terms of efficacy outcomes, Groot and colleagues derived mean disease-free survival through summing and calculating data estimated from the previously-mentioned databases. QALYs were based on a study [24] which only looked at elderly NHL patients, even though younger patients were also included by Groot and colleagues. Using weighted averages of the utilities, the authors assigned scores based on initial treatment $(0.60)$, progression-free disease $(0.81)$, disease progression (0.60), or death (0.00). Since the same utility scores were assumed for CHOP and R-CHOP, the outcome measure of QALYs would specifically reflect the number of years spent in any of the four utility categories for either of the two treatment arms.

Results found by Groot and colleagues were divided between younger and older patients. After discounting, younger patients (age $<60$ years) were found to have a gain in 0.88 QALYs by receiving R-CHOP versus CHOP-only therapy. However, costs in this patient group were also higher when receiving $\mathrm{R}$-CHOP $(€ 12,343$ more in total costs versus CHOP-only). The incremental ratio derived for younger patients was therefore $€ 13,983 / \mathrm{QALY}$ through use of R-CHOP versus CHOP-only. A similar trend was seen in the older patient population, where the incremental ratio was $€ 17,933 / \mathrm{Q} A L Y$ through use of R-CHOP versus CHOP-only. Thus, R-CHOP therapy was found to be cost-effective in terms of QALYs gained by keeping in mind the accepted threshold of $€ 50,000$.

Groot and colleagues performed numerous one-way sensitivity analyses to verify the robustness of the baseline results. A shortened time horizon of only five years was found to have the most profound impact, pushing the incremental ratio to $€ 35,355$ for older patients. Still, this value is below the internationally accepted threshold of $€ 50,000$.

Another cost-utility analysis by Best and colleagues [25] used a similar time horizon of fifteen years and reported values in QALYs. Like the previous study by Groot and colleagues, Best and colleagues also modeled event probabilities using data from previously-published trials, including long-term mortality rates based on the Scottish Newcastle Lymphoma Group (SNLG) data. The authors stated a payer perspective, including direct medical costs such as drug costs, surveillance, hospitalizations due to adverse events, high-dose rescue therapy, and hospice care. The study did not include stem-cell transplantation costs, although this was accounted for in sensitivity analyses.

Results from the study showed that total costs discounted $3 \%$ in the CHOP-only treatment arm were lower $(€ 28,782)$ than that in the R-CHOP treatment arm $(€ 41,952)$ mainly due to the added drug cost of rituximab. However, the CHOP-only group demonstrated 3.59 QALYs whereas RCHOP resulted in 4.66 QALYs. Thus, the incremental ratio projected at fifteen years was favorable at $€ 12,259 / \mathrm{QALY}$ with use of R-CHOP versus CHOP-only.

Best and colleagues also investigated the robustness of the baseline results through numerous sensitivity analyses, showing that a varied time horizon had the most impact on results. Shortening the time horizon to only four years caused the incremental ratio for $\mathrm{R}-\mathrm{CHOP}$ to rise to $€ 29,976 / \mathrm{QALY}$, although clearly still within the acceptable threshold. A thorough investigation of other variables, such as cost of drugs, discount rates, and event probabilities all confirmed the robustness of the baseline results with incremental ratios within the acceptable threshold.

Finally, Hornberger and colleagues [26] similarly investigated the incremental cost-utility of R-CHOP versus CHOP-only regimens in elderly patients with DLBCL. The stated perspective of the study was from the US societal perspective. Like the other reviewed studies, event probability data was derived from previously-published studies, such as the Group d'Etude des Lymphome d'Adulte (GELA) Trial [27]. Since the GELA Trial was performed in Europe, the authors derived costs from U.S. formulary services and U.S.based literature. Included costs were drug costs, posttreatment surveillance costs (e.g. laboratory investigations), rescue therapy costs, stem-cell transplantation costs, and hospice care costs. A 3\% discount rate was used. 
A time horizon of five years was used in the study. Utilities were assigned to the categories of event-free survival (0.83), end-of-life (0.38), and salvage or transplantation (0.83) for the R-CHOP and CHOP-only treatment arms. Assigned utilities were derived from published literature [28] involving CHOP therapy in NHL patients, the same utility data used for the other reviewed studies. Baseline results revealed that the incremental cost-utility ratio for R-CHOP was \$19,297/QALY gained.

Sensitivity analyses conducted by Hornberger and colleagues confirmed the robustness of the baseline case. Like the other reviewed studies, time horizon seemed to have the most impact on resultant incremental ratios. Shortening the time horizon to three years caused the ratio to increase to $\$ 46,942$ per 1 QALY gained with R-CHOP versus CHOPonly treatment. Other variables, such as cost of chemotherapy agents, discounting rate, cost of surveillance, cost of hospice care, and cost of transplantation upheld the robustness of the baseline case with ratios well below the acceptable threshold of $\$ 50,000$.

\section{DISCUSSION}

The pharmacoeconomic methods employed in the reviewed studies showcase how fundamental economic analyses can augment clinical decision-making efforts. While the four reviewed studies are generally in agreement in terms of demonstrating the acceptable cost-effectiveness and costutility of R-CHOP over conventional CHOP therapy, it is important to note the effects of proper (or improper) implementation of basic pharmacoeconomic principles.

One of the first principles of a pharmacoeconomic analysis is to understand from which perspective the analysis is being done. It is possible for a study to be done from the perspective of the patient, the provider, the employer, the payer, or from the perspective of society as a whole. Depending on which perspective is taken, differing costs may be included. For instance, if a payer perspective is stated, then only those costs applicable from the payer's point of view would be included. A payer, such as a national health service, would be interested in only direct medical costs, as these are the only costs the payer is responsible for. The reviewed study by Ferrara and colleagues, for example, stated a payer perspective of the Italian National Health Service. While the authors do clearly state several appropriate direct medical costs for inclusion (chemotherapy drugs, hematological investigations, laboratory investigations, imaging, histological analyses, stem-cell transplantation, and hospitalization costs), it must be pointed out that the authors failed to include other relevant direct medical costs, such as nursing administration costs, pharmacists' dispensing fees, and costs derived from potential adverse reactions to the regimens given. Including an exhaustive list of all costs relevant to the payer allows for a more accurate picture of all costs incurred by the treatment(s). Klepser [29] points out that including only straightforward costs - such as drug and biochemical investigation costs - can severely underestimate the true costs incurred.

It was found that the other reviewed studies also fell victim to similar pitfalls. Groot and colleagues and Hornberger and colleagues both stated societal perspectives, yet included only direct medical costs. Groot and colleagues in fact only considered wholesale drug costs and specialist visits, while Hornberger and colleagues investigated drug costs, posttreatment surveillance costs, rescue therapy costs, transplantation costs, and hospice care costs. Both studies instead would more accurately be stated to have taken on a payer perspective since only direct medical costs have been included. A truly societal perspective would have included monetary amounts associated with indirect medical costs as well as intangible costs.

Beyond creating an accurate picture of all incurred costs according to the perspective taken, sound use of pharmacoeconomic principles would also comprise proper outcomes measures. For instance, it would be inappropriate to measure life years gained (LYG) in a study investigating a disease where patients still live a relatively full lifespan. It likewise would be inappropriate to mismatch outcomes, such as measuring outcomes in terms of symptom-free days in a relatively silent disease state like early hypertension. A strength found in the reviewed studies is that both costeffectiveness and cost-utility analyses provide the means to evaluate outcomes accurately in the case of NHL. For instance, Ferrara and colleagues used cost-effectiveness analysis to look at clinical outcomes in terms of life years gained (LYG). Hornberger and colleagues likewise used cost-utility analysis to investigate humanistic outcomes in terms of quality-adjusted life years (QALYs). Both methods allowed researchers to test the dominance of the treatment in question for example, whether or not R-CHOP was dominant over CHOP-only in terms of lower costs and greater outcomes. In the cases where R-CHOP was higher in cost, both analytical methods allowed outcomes to be reported in incremental ratios. The outcomes measures used in the reviewed studies were applicable because LYG and QALYs are often used to measure other well-studied cancer-related outcomes [30, 31]. A strength, therefore, found in all of the reviewed studies was that appropriate measures of outcomes were used - survival in terms of years of life gained is often the most-sought outcome in cancer research. Further, the incorporation of quality of life through use of QALYs has emerged as an especially suitable endpoint in cancer studies [32].

Finally, it is important to realize that even with careful concern for the appropriate inclusion of all costs and outcomes, there is still a level of uncertainty when dealing with any set of data. Sensitivity analyses or "what if" analyses are vital to any type of pharmacoeconomic evaluation as they are a way to transparently examine the imprecision or uncertainty of the data used. In essence, these analyses allow investigators to test the robustness of baseline results using ranges of reasonable data that may or may not have affected the drawn conclusions. Including comprehensive sensitivity analyses in a study allows greater practicality when interpreting the results in real-world situations. One of the simplest ways to test the robustness of a study's results is to run a series of sensitivity analyses on any uncertain data inputted in the study, such as drug costs, disease risk or event probabilities, assumed survival rates, weighting of qualities, discount rates, and all other imprecise variables [33].

The reviewed studies have demonstrated how use of sensitivity analyses can support the robustness of the baseline 
results. The sensitivity analyses used by Ferrara and colleagues investigated the impact of using worst-case scenario event probabilities for the assumed survival and response rates. Incremental cost-effectiveness ratios were confirmed to be below the acceptable threshold of $€ 50,000$ for the varied complete response at five months and relapse-free survival at three years. However, the authors did not test variables such as acquisition prices of drugs which would have further added to the validity of the study. The use of price ranges, for instance $+/-20 \%$ of each assumed drug cost, would have allowed the authors to test the role varying drug costs played in the results of the study. Briggs et al. [34] emphasizes the importance such multivariate sensitivity analyses in the generalizability of study results, pointing out that the cost-effectiveness found in one trial may not be applicable to another practice setting.

The reviewed studies largely illustrated the impact sensitivity analyses can have on the baseline results of cost-utility analyses. Groot and colleagues found that an incremental ratio of $€ 17,933 / \mathrm{QALY}$ was gained through use of R-CHOP versus $\mathrm{CHOP}$-only in their 15 -year baseline results. Following a sensitivity analysis of a shortened time horizon of five years, the ratio nearly doubled to $€ 35,355 / \mathrm{Q} A L Y$ gained. Sensitivity analyses by Best and colleagues and Hornberger and colleagues illustrated similar results. Hornberger and colleagues reported that a shortened time horizon caused the incremental cost-utility ratio to increase to $\$ 46,942 / \mathrm{QALY}$ gained with R-CHOP versus CHOP-only treatment (baseline $=\$ 19,297 /$ QALY gained). Thus, the reviewed studies offer a profound picture of how sensitivity analyses can alter the baseline results. While each of the studies' sensitivity analyses upheld the baseline cost-effectiveness or cost-utility of $\mathrm{R}-\mathrm{CHOP}$, the profound increase in incremental costs per outcome gained is extremely relevant in terms of clinical decision making. As the incremental ratios approach the threshold of 50,000 in international monetary amounts, clinical decision-makers are faced with tougher decisions about allocation of health care dollars. Without the strength of such sensitivity analyses, this impact would not be seen.

As this review has demonstrated, pharmacoeconomic evaluations are multi-faceted and can be diverse in terms of substance and quality. Reviewing and subsequently comparing multiple health care studies can be overwhelming to even the most organized decision maker. Fortunately, there exists a useful tool for comparing the quality of health care economics studies in a concise side-by-side fashion. The Quality of Health Economic Studies (QHES) instrument was developed to address the major evaluative techniques of a quality health economic study. The QHES tool is an invaluable organizational tool for savvy pharmacoeconomic reviewers and the checklist provides an all-inclusive method to assigning a "rank" to multiple studies being considered by a health care decision maker. Doan et al. [35] have demonstrated the value of adopting the QHES checklist in their review of eight pharmacoeconomic studies.

The four pharmacoeconomic studies included in this review have been analyzed using an adaptation of the QHES instrument (Table 3). Since the tool allows a quantitative "ranking" of the reviewed studies, health care decision mak- ers can quickly filter through a plethora of published pharmacoeconomic studies and focus on those ranked most comprehensive in accordance with the demonstrated value of the QHES. One limitation, naturally, is that the designation of the qualifying points is based on the designator's opinion and critique of the reviewed study. In other words, the point values awarded to the studies are subject to the reviewer's own judgments and interpretation of the QHES tool.

For purposes of this review, points were awarded in an "all or none" fashion, meaning that if the reviewer felt that the study did not fully meet the standards of each of the sixteen criteria, a zero-point value was awarded. For example, while Ferrara and colleagues do include a measurement of costs and methodology for the estimation of quantities and unit costs, this review points out that an entirely comprehensive inclusion of all costs relevant to the payer perspective was not included. Thus, the study by Ferrara and colleagues was assigned a zero-point value for criterion 9, as were all other reviewed studies using the same rationale. Likewise, a zero-point value was awarded if the study was found lacking in a criterion or did not accurately fulfill the requirements of a criterion. For example, while Ferrara and colleagues clearly state the intended perspective of their pharmacoeconomic study, their subsequent inclusions of costs do not wholly fulfill the requirements of a payer perspective. For this reason, a zero-point value was awarded to the Ferrara and colleagues for criterion 2. In the same regard, Groot and colleagues and Hornberger and colleagues also were award zero points for criterion 2 because they purported a societal perspective but included only direct medical costs. While all four reviewed studies included sensitivity analyses, the study by Ferrara and colleagues was awarded a zero-point value for criterion 5 due to the judgment that its efforts at multiple sensitivity analyses lacked when compared to the other reviewed studies in terms of range and scope. On the other hand, the study by Ferrara and colleagues was judged superior compared to the other reviewed studies in terms of transparency of incremental analyses and explanation of methodology; thus, the study was alone awarded a point value of eight for criterion 12 . The study by Groot and colleagues alone failed to clearly disclose a funding source and therefore was the only reviewed study awarded a zero-point value for criterion 16. This review also found that none of the reviewed studies adequately explored potential biases in an explicit manner, so zero-point values were awarded across the board. It must be noted that judgments formed through this type of ranking are subject to opinion and must be considered when interpreting the QHES checklist.

In conclusion, while there are limited pharmacoeconomic studies available in the specific arena of R-CHOP use in non-Hodgkin lymphoma patients, the reviewed studies suggest agreement that R-CHOP incurs acceptable costs per outcomes over CHOP-only in the studied patient population. All of the reviewed studies shared the common weakness of not including a proper, comprehensive list of incurred costs for the perspectives stated. Thus, it can be ascertained that while the available studies provide a foundation for future pharmacoeconomic studies, one must be careful in interpreting the external validity of the currently reviewed evaluations. Since all of the reviewed studies lacked proper inclusion of costs, clinical decision makers must be wary in ap- 
Table 3. The Quality of Health Economic Studies Instrument Adapted for the Review of R-CHOP Versus CHOP-alone Analyses*

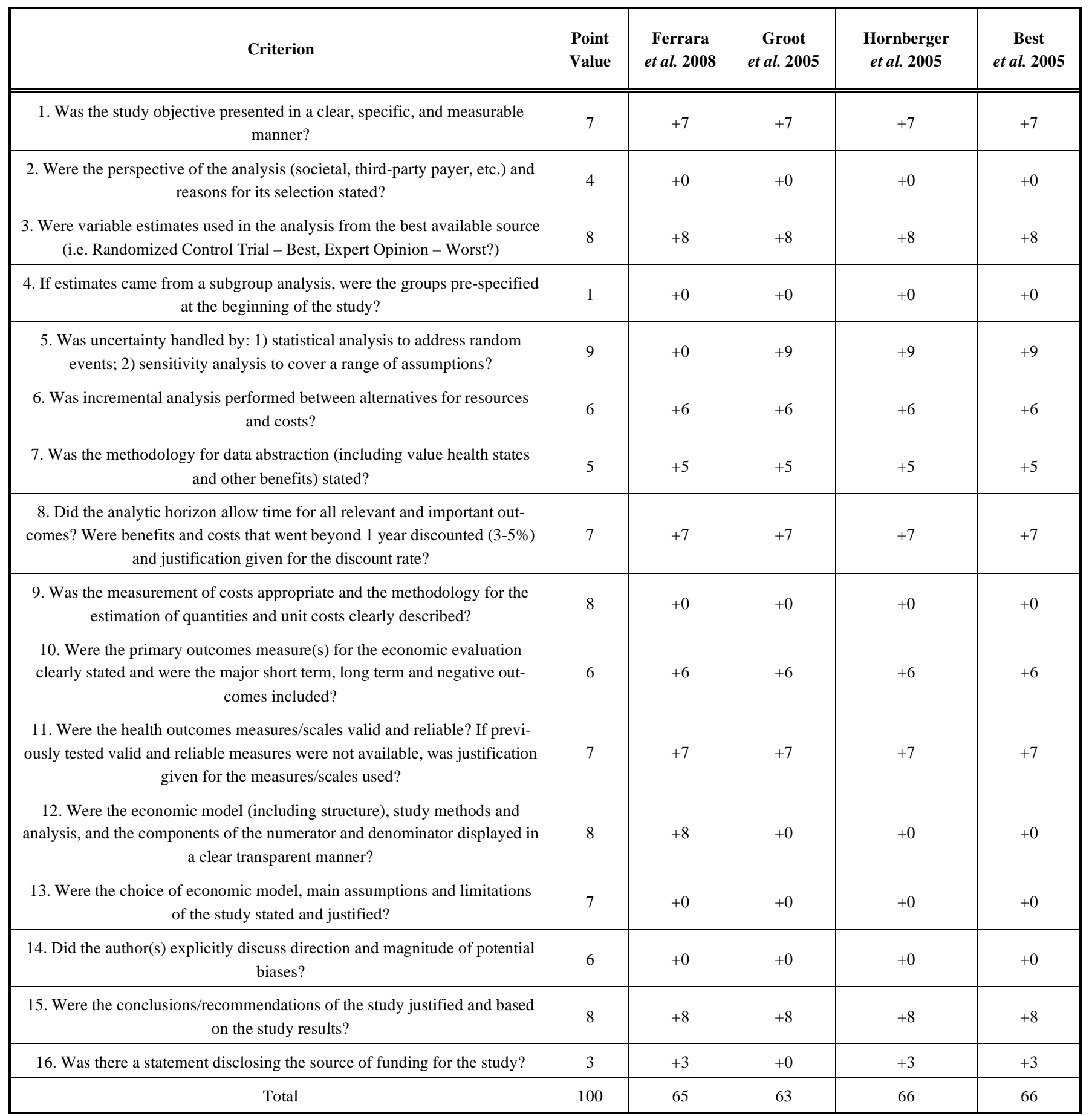

*Adapted from The QHES Instrument presented by Ofman and colleagues [14].

plying the same results to their own institutions. The most profound impact found in the pharmacoeconomic review of R-CHOP versus CHOP-only related to study time horizon. Without the sensitivity analyses done by the included studies with regard to a shortened time horizon, the transparency of the results would have been lost. Thus, clinical decisionmakers must take seriously the implications of sensitivity analyses; as the reviewed studies suggest that incremental cost-utility of R-CHOP is diminished as the time horizon shortens. A lesson learned by the limited studies reviewed here is that the results of a study can only be assured for the circumstances used in the study. When attempting to extrapolate results of a pharmacoeconomic study to one's own practice, it is vital to take into account one's own incurred costs relevant to that perspective. Also, the thorough use of sensitivity analyses is demanded as all inputted data and costs are subject to uncertainty. 


\section{LIST OF ABBREVIATIONS}

$\begin{array}{lll}\text { CHOP } & = & \begin{array}{l}\text { Cyclophosphamide/doxorubicin/ } \\ \text { vincristine/prednisone }\end{array} \\ \text { R-CHOP } & = & \begin{array}{l}\text { Rituximab plus Cyclophosphamide/ } \\ \text { doxorubicin/vincristine/prednisone }\end{array} \\ \text { QALYs } & = & \text { Quality-adjusted life years } \\ \text { HL } & = & \text { Hodgkin lymphoma } \\ \text { NHL } & = & \text { Non-Hodgkin lymphoma } \\ \text { DLBCL } & = & \text { Diffuse large-B-cell lymphoma } \\ \text { MInT } & = & \text { MabThera International Trial } \\ \text { QHES } & = & \text { Quality of Health Economic Studies } \\ \text { LYG } & = & \text { Life years gained } \\ \text { GELA } & = & \text { Group d'Etude des Lymphome d'Adulte }\end{array}$

\section{REFERENCES}

[1] Mittala A, Marshallb NA, Barkerb RN, Vickers MA. Immunomodulation against leukemias and lymphomas: A realistic future treatment. Crit Rev Oncol Hematol 2008; 65(2): 101-8.

[2] American Cancer Society. Monoclonal Antibodies. 14 Dec 2006. Available at: http://www.cancer.org/docroot/ETO/content/ETO_1_4X_Monoclo nal_Antibody_Therapy_ Passive_Immunotherapy.asp. Accessed March 27, 2008.

[3] Lymphoma Research Foundation. About Lymphoma. Available at: http://www.lymphoma.org/site/pp.asp?c=chKOI6PEImE\&b=15732 89. Accessed March 27, 2008

[4] Maloney D, Kahl BS, Dreyling M. Indolent lymphomas: current and emerging treatment approaches. Clin Adv Hematol Oncol 2006; 4(9 Suppl 20): 1-10.

[5] National Cancer Institute. Non-Hodgkin Lymphoma. Available at: http://www.cancer.gov/cancertopics/types/non-hodgkin. Accessed March 27, 2008.

[6] UpToDate. Lymphoma. Available at: http://www.uptodate.com. Accessed March 27, 2008.

[7] Marcus R, Hagenbeek A. The therapeutic use of rituximab in nonHodgkin's lymphoma. Eur J Haematol Suppl 2007; 67: 5-14.

[8] Coiffier B, Lepage E, Briere J, et al. CHOP chemotherapy plus rituximab compared with CHOP alone in elderly patients with diffuse large-B-cell lymphoma. N Engl J Med 2002; 346: 235-42.

[9] Pfreundschuh M, Trumper L, Osterborg A, et al. CHOP-like chemotherapy plus rituximab versus CHOP-like chemotherapy alone in young patients with good-prognosis diffuse large- B-cell lymphoma: a randomized controlled trial by the MabThera International Trial (MInT) Group. Lancet Oncol 2006; 7(5): 379-91.

[10] Schulz H, Bohlius J, Skoetz N, et al. Chemotherapy plus rituximab versus chemotherapy alone for B-cell non-Hodgkin's lymphoma. Cochrane Database Syst Rev 2007; Issue 4.

[11] Reeder CE. Symposium: Overview of pharmacoeconomics and pharmaceutical outcomes evaluations. Am J Health Syst Pharm 1995; 52(19): 5s-8s.

[12] Phillips C, Thompson G. What is cost-effectiveness analysis? Bandolier Extra 2001; 1(3): 1-6.

[13] Chiou CF, Hay JW, Wallace JF, et al. Development and validation of a grading system for the quality of cost-effectiveness studies. Med Care 2003; 41(1): 32-44.

[14] Ofman JJ, Sullivan SD, Neumann PJ, et al. Examining the value and quality of health economic analyses: implications of utilizing the QHES. J Manag Care Pharm 2003; 9(1):53-61.
[15] Rothermich EA, Pathak DS. Productivity-cost controversies in cost-effectiveness analysis: review and research agenda. Clin Ther 1999; 21(1): 255-67.

[16] Eichler HG, Kong SX, Gerth WC, et al. Use of cost-effectiveness analysis in health-care resource allocation decision-making: how are cost-effectiveness thresholds expected to emerge? Value Health 2004; 7(5): 518-29.

[17] Gan TJ, Lubarsky DA. Cost-effectiveness ratio: an often misunderstood term. Anesth Analg 1999; 88: 1191-2.

[18] Detsky AS, Naglie G, Krahn MD, et al. Primer on Medical Decision Analysis - Part I: Getting Started. Med Decis Making 1997; 17(2): $123-5$

[19] Ferrara F, Ravasio R. Cost-effectiveness analysis of the addition of rituximab to $\mathrm{CHOP}$ in young patients with good-prognosis diffuse large-B-cell lymphoma. Clin Drug Investig 2008; 28(1): 55-65.

[20] Joish VN, Oderda GM. Cost-utility analysis and quality adjusted life years. J Pain Palliat Care Pharmacother 2005; 19(1): 57-61.

[21] Dolan P. Valuing health-related quality of life: issues and controversies. Pharmacoeconomics 1998; 15(2): 119-27.

[22] Groot MT, Lugtenburg PJ, Hornberger J, et al. Cost-effectiveness of rituximab (MabThera) in diffuse large B-cell lymphoma in The Netherlands. Eur J Haematol 2005; 74(3): 194- 202.

[23] Agthoven M, Faber LM, Uyl-de Groot P, et al. Cost analysis of CHOP-like chemotherapy regimens for patients with newly diagnosed aggressive non-Hodgkin's lymphoma. Eur J Haematol 2002; 69(4): 213-20.

[24] Doorduijn J, Buijt I, van der Holt B, et al. Self-reported quality of life in elderly patients with aggressive non-Hodgkin's lymphoma treated with CHOP chemotherapy. Eur J Haematol 2005; 75: 11623.

[25] Best JH, Hornberger J, Proctor SJ, et al. Cost-effectiveness analysis of rituximab combined with CHOP for treatment of diffuse large Bcell lymphoma. Value Health 2005; 8(4): 462- 70.

[26] Hornberger JC, Best JH. Cost utility in the United States of rituximab plus cyclophosphamide, doxorubicin, vincristine, and prednisone for the treatment of elderly patients with diffuse large B-cell lymphoma. Cancer 2005; 103(8): 1644-51.

[27] DLBCL LNH 98-5 Phase III Groupe d'Etude des Lymphomas de a'Adulte (GELA) Trial. Available at: http://www.rituxan.com/ lymphoma/HCP/Efficacy/GELA-LNHSummary.jsp. Accessed, April 3, 2008.

[28] Doorduijn J, Buijt I, van der Holt B, et al. Self-reported quality of life in elderly patients with aggressive non-Hodgkin's lymphoma treated with CHOP chemotherapy. Eur J Haematol 2005; 75: 11623.

[29] Klepser D. Pitfalls associated with commonly used methods for pharmacoeconomic analyses. Pharmacotherapy 2002; $22(22$ Suppl.): $35 \mathrm{~s}-38 \mathrm{~s}$.

[30] Reeder C, Gordon D. Managing oncology costs. Am J Manag Care. 2006; (Suppl. 12): 3-16.

[31] Karnon J, Johnston S, Jones $\mathrm{T}$, et al. A trial-based costeffectiveness analysis of letrozole followed by tamoxifen versus tamoxifen followed by letrozole for postmenopausal advanced breast cancer. Ann Oncol 2003; 4(11): 1629-33.

[32] Steer CB, Marx GM, Galani E, Harper PG, Khayat D. Quality of life: it's never too late. Am J Clin Oncol 2002; 20(13): 2915-17.

[33] Hay JW. Economic modeling and sensitivity analysis. Value Health 1998; 1(3): 187-93.

[34] Briggs A, Sculpher M, Buxton M. Uncertainty in the economic evaluation of health care technologies: the role of sensitivity analysis. Health Econ 1994; 3: 95-104.

[35] Doan QV, Chiou CF, Dubois RW. Review of eight pharmacoeconomic studies of the value of biologic DMARDs (adalimumab, etanercept, and infliximab) in the management of rheumatoid arthritis. J Manag Care Pharm 2006; 12(7): 555-569.

(C) Yoder and Kamal; Licensee Bentham Open.

This is an open access article licensed under the terms of the Creative Commons Attribution Non-Commercial License (http://creativecommons.org/licenses/by-nc/3.0/) which permits unrestricted, non-commercial use, distribution and reproduction in any medium, provided the work is properly cited. 